\title{
Offline Impairment Aware RWA Algorithms for Cross-Layer Planning of Optical Networks
}

\author{
Pablo Pavon-Mariño, Siamak Azodolmolky, Ramon Aparicio-Pardo, Belen Garcia-Manrubia, Yvan \\ Pointurier, Marianna Angelou, Josep Solé Pareta, Joan Garcia Haro, Ioannis Tomkos
}

\begin{abstract}
Transparent optical networks are the enabling infrastructure for converged multi-granular networks in the Future Internet. The cross-layer planning of these networks considers physical impairments in the network layer design. This is complicated by the diversity of modulation formats, transmission rates, amplification and compensation equipments, or deployed fiber links. Thereby, the concept of Quality of Transmission (QoT) attempts to embrace the effects of the physical layer impairments, to introduce them in a multicriterium optimization and planning process. This paper contributes in this field by the proposal and comparative evaluation of two novel offline impairment aware planning algorithms for transparent optical networks, which share a common QoT evaluation function. The first algorithm is based on an iterative global search driven by a set of binary integer linear programming formulations. Heuristic techniques are included to limit the binary programming complexity. The second algorithm performs different pre-orderings of the lightpath demand, followed by a sequential processing of the lightpath demands. The performance and the scalability of both approaches are investigated. Results reveal great scalability properties of the global search algorithm, and a performance similar to or better than the sequential schemes.
\end{abstract}

Index Terms-Physical layer Impairments Aware, Routing and Wavelength Assignment, Optical Network Planning

Manuscript received December 15, 2008. The work described in this paper was carried out with the support of the BONE project ("Building the Future Optical Network in Europe"); a Network of Excellence, and the DICONET project ("Dynamic Impairment Constraint Networking for Transparent Mesh Networks"), both funded by the European Commission through the 7th ICTFramework Program.

This research has been partially supported by the MEC project TEC200767966-01/TCM CON-PARTE-1, and it is also developed in the framework of "Programa de Ayudas a Grupos de Excelencia de la R. de Murcia, F. Séneca".

Pablo Pavon Marino, Ramon Aparicio Pardo, Belen Garcia Manrubia and Joan Garcia Haro are with Universidad Politécnica de Cartagena (UPCT), Plaza Hospital 1, 30202, Cartagena, Spain (phone: +34 968325952; fax: +34 968325973; e-mail: pablo.pavon@upct.es, \{rap,mbgm\}@alu.upct.es, joang.haro@upct.es).

Siamak Azodolmolky and Josep Solé Pareta are with the Technical University of Catalonia (UPC), C/Jordi Girona, 1-3. 08034 Barcelona, Catalonia, Spain (phone: +30-210-6682792; fax: +30-210-6682729; e-mail: \{siamak,pareta\}@ac.upc.edu).

Yvan Pointurier, Marianna Angelou and Ioannis Tomkos are with Athens Information Technology, 0.8km Markopolou Ave., Peania 19002, Athens, Greece (e-mail: \{yvan, mang, itom\}@ait.edu.gr).

\section{INTRODUCTION}

$\mathrm{T}$ ransparent optical networks have been proposed to decrease costs and increase capacity in the future generation, so-called "converged" network architectures [1], [2]. In transparent optical networks, traffic is carried onto lightpaths which occupy one transmission wavelength in each traversed link. The carried traffic is processed electronically at the ingress and egress nodes of the lightpath, but not at the intermediate transit nodes, saving electronic switching costs, and providing the data plane with a traffic format transparency. From a network planning perspective, the problem of finding the lightpaths to accommodate a given traffic demand is called the routing and wavelength assignment (RWA) problem [3], [4], [5], [6], [7], [8].

Transparency, however, comes at a cost: lightpaths can be very long and physical layer impairments can accumulate and unacceptably decrease transmitted signals' Quality of Transmission (QoT, usually measured in terms of Bit-Error Rates, BER). Should the QoT of a lightpath drop beyond a predetermined threshold, then this lightpath would be blocked, an event called "QoT blocking". For this reason, cross-layer planning techniques are needed in transparent networks to introduce physical layer considerations in the network layer design. Moreover, in a converged network infrastructure, multiple protocols and transport technologies are expected to coexist. The cross-layer optimization is then hindered by the diversity of modulation formats, transmission rates, and a plethora of optical equipments in the network.

In this scenario, the multi-criteria planning of transparent optical networks has recently received much attention from the research community [1], [5], [9]. This paper addresses the planning of future generation transparent optical networks through PLIA-RWA (Physical Layer Impairment Aware RWA), a cross-layer technique. PLIA-RWA incorporates several constraints (limited amount of resources and imperfect physical layer) and hence solves a central planning problem in converged optical networks.

Two main types of optimization strategies exist when dealing with the network planning process. They are commonly called as offline and online planning. In offline planning, the traffic demand is assumed as fixed and known in advance, while online planning designs the reactions to the arrival of lightpath establishment requests along time. Thus, 
the online planning problem is commonly addressed by processing individual requests one by one, examining the feasibility of the possible lightpaths for each connection request. In fact, in the online planning scenario we can always calculate (through appropriate analytical models) or measure (by optical performance/impairment monitoring) the interference of the other channels to the lightpath under investigation, since other lightpaths have already been established when the algorithm is executed. Both online and offline approaches are relevant in a converged network infrastructure. A fraction of the total traffic demand is expected to be predictable background traffic, appropriately planned with offline techniques, while the remaining traffic demand is expected to be dynamic, planned by means of online techniques.

Most of the algorithms proposed in the open literature address the online version of the PLIA-RWA problem. A comprehensive review is compiled in [9]. In contrast to this, the investigations for PLIA-RWA offline planning are less. This is mainly due to the fact that even the classical offline RWA problem is NP-complete, and it becomes more complicated when considering signal impairments, which imply cross-layer optimization techniques. Then, planning algorithms have to make use of (i) specific functions that estimate the QoT for the lightpaths in a given virtual topology, and (ii) an optimization core which intends to explore the solutions space smartly.

This paper presents and compares two novel algorithms suitable for the offline PLIA-RWA problem. Our algorithms make use of a common QoT estimation function named $Q$ tool, also developed by the authors. No wavelength conversion equipment is assumed in the network. The $Q$-tool function is able to calculate the so-called $Q$-factor of the lightpaths in a virtual topology. The $Q$-factor of a lightpath is in direct relation to its signal Bit Error Rate (BER) performance [10]. The first proposed algorithm heuristically combines a set of Binary Integer Linear Programming (BILP) formulations. It is designed to allow a global search of the solutions space, but limiting the complexity of the BILP formulations to guarantee its scalability. As far as the authors know, this is the first proposal following this approach. Then, two variations of a heuristic algorithm are proposed which explore the solutions space by first sequencing the traffic demand in different manners, and then processing the demands one by one. The proposed schemes are compared in different scenarios, evaluating their lightpath blocking rate performance. Also, the scalability of the algorithms is assessed by evaluating the algorithms' response time in diverse situations.

The rest of the paper is organized as follows. In Section II, a review on existing offline PLIA-RWA algorithms is provided. Section III presents the QoT performance evaluator ( $Q$-tool) developed. Section IV presents our PLIA-RWA algorithm based on the global optimization approach and is followed by the traffic demand sequencing approach in Section V. Comparative simulation results are compiled in
Section VI. The study on the scalability concerns of the algorithms is included in Section VII. Finally, Section VIII concludes.

\section{STATE-OF-THE-ART IN OFFLINE PLIA-RWA ALGORITHMS}

This section surveys a set of relevant contributions in the field of offline PLIA-RWA planning. A comprehensive review for the offline case (as well as the online case) can be found in [9].

In [11] a link-path Integer Linear Programming (ILP) formulation for the classical RWA problem is proposed. Impairment constraints are taken into account by appropriately pre-processing the data which feed the RWA formulation. This is done by pre-calculating a set of $k$ paths with the assistance of a shortest path algorithm, which uses either a single physical impairment [11] or a $Q$-penalty [12] as the link cost parameter. Then, the virtual topology is calculated by the ILP formulation, considering only the set of candidate paths. Finally, the virtual topology resulting from the RWA formulation is post-processed by evaluating the QoT feasibility of its lightpaths. For the lightpaths with unacceptable transmission performance, new solutions are searched by excluding lightpaths that were previously considered from the original set of candidate paths.

An impairment-aware offline RWA algorithm that assigns $Q$-factor costs to the links before solving the problem is proposed in [13]. In this work, $k$-shortest routes are computed considering $Q$-penalty values as the links costs. Finally the wavelength that maximizes the $Q$ value is selected for each connection request. Since the wavelength assignment is not performed jointly for all connections, a worst case scenario for the interference among lightpaths is used. Therefore, the proposed algorithm does not take into account the interference among lightpaths and does not optimize the solution in order to avoid inter-lightpath interferences.

Some more specific proposals that introduce physical layer impairment constraints into the optimization problem were studied as well. A Mixed Integer Linear Programming (MILP) formulation for the RWA problem of multicast connections, while considering optical power constraints, is presented in [14]. The authors formulate the RWA problem considering the optical power in order to ensure that the power level at the beginning of each optical amplifier, and also at the end of each fiber is above a certain threshold.

An ILP formulation for the problem of traffic grooming in optical virtual private networks with the BER constraint is presented in [15]. The physical layer impairments and the BER are indirectly taken into account in the ILP formulation through the length of the path.

In [16] the implementation of an LP solver in a Path Computation Element (PCE) is reported. The implemented objective function minimizes the maximum link bandwidth utilization. As a result the routes satisfying the required constraint in terms of bandwidth and optical signal quality can be found. However, it should be noted that it does not propose 
an algorithm but a general architecture to consider physical impairments in a Path Computation Element (PCE).

Finally, it is worth mentioning that online algorithms can be used to solve the offline problem. This can be done by considering the offline connections sequentially and serve them on a one-by-one basis. In order to use an online algorithm for solving the offline problem the algorithm has to be able to take into account the effect of the existing connections [17]. In general, these approaches do not optimize the utilization of wavelengths for all connections requests jointly and thus their performance is suboptimal.

When online algorithms are adapted for the offline case, the order in which the connections are served is flexible and particularly important for the final solution. Therefore, to improve the performance, an ordering phase can be used before sequentially serving the connection requests. Another approach that can enhance the performance of such schemes is re-routings. Re-routing refers to the re-computation and reestablishment of already established connections when a new lightpath is considered.

The work presented in this paper contributes to the offline PLIA-RWA planning field in several forms. First, as far as the authors know, the global searching algorithm proposed is the first attempt to heuristically combine BILP formulations for the PLIA-RWA problem, in a manner which limits the complexity to guarantee algorithm scalability at common network sizes. In addition, a family of algorithms is proposed to investigate the benefits of optimization techniques which pre-orders the traffic demand following some suitable criteria, and processes each connection request sequentially. All approaches share a common $Q$-factor evaluation function, to make a fair comparison and extract useful conclusions about the most suitable optimization approaches. Also, the scalability of the algorithms is studied.

\section{III. $Q$-TOOL: PhysicAl LAYER PERformance EVAluator}

In the context of transparent networks, impairments can be categorized into static and dynamic ones. Static impairments are topology-dependent, and independent from the routing state of the network. The static impairments considered in this work are Amplifier Spontaneous Emission (ASE) noise, filter concatenation, and Polarization Mode Dispersion (PMD). Self Phase Modulation (SPM) (and its interaction with chromatic dispersion) was not included because of its negligible contribution (as indicated in [18]) and its static nature that renders it independent of the traffic. Dynamic impairments depend on the presence and characteristics of other lightpaths already established in the network. This work accounts for the following dynamic impairments: node crosstalk, originating from signal leaks at nodes, and nonlinear effects: Cross Phase Modulation (XPM) and Four Wave Mixing (FWM).

The Quality of Transmission (QoT) of a lightpath is evaluated by the same own developed tool named $Q$-tool. $Q$ tool computes the so-called $Q$-factor of the lightpaths of a virtual topology, given the physical characteristics of the network. The $Q$-factor for a lightpath is a QoT indicator which is related to the signal's Bit-Error Rate (BER). For an On-Off modulated signal the following relation is used:

$B E R=\frac{1}{2} \operatorname{erfc}\left(\frac{Q}{\sqrt{2}}\right)$

The Q-factor is defined as reference [10]:

$Q=\frac{P_{1}-P_{0}}{\sigma_{0}+\sigma_{1}}$

where $P_{1}$ and $P_{0}$ are the means of the distributions (assumed to be Gaussian) of the received samples corresponding to sent "1" and " 0 " bits, and $\sigma_{1}$ and $\sigma_{0}$ are the respective standard deviations. We consider that $P_{0}$ is negligible compared with $P_{1}$. Although the Gaussian assumption may not always be accurate, there is always a strong correlation between $Q$ factor and BER and hence we use $Q$ factor as a Quality of Transmission estimator to represent BER performance. To estimate a $Q$-factor, the $Q$-tool actually computes the following quantity:

$Q=\frac{\eta_{P M D} P_{1}^{\prime}}{\sigma_{0}+\sigma_{1}}$

As suggested in [19], we model filter concatenation impairment as an eye closure penalty, yielding the term $P_{1}$ '. The PMD effect is modeled as a penalty on the $Q$-factor as in [20] through the multiplicative factor $\eta_{P M D}$.

Other impairments are accounted for through noise variances:

$\sigma_{1}^{2}=\sigma_{1, A S E}^{2}+\sigma_{1, X T}^{2}+\sigma_{1, X P M}^{2}+\sigma_{1, F W M}^{2}$,

$\sigma_{0}^{2}=\sigma_{0, A S E}^{2}+\sigma_{0, X T}^{2}$.

ASE noise is modeled as a noise variance according to [10] and contributes to both $\sigma_{1}$ and $\sigma_{0}$ via $\sigma_{1, A S E}^{2}$ and $\sigma_{0, A S E}^{2}$, respectively. Since $P_{1}, \quad \sigma_{1, A S E}^{2}$ and $\sigma_{0, A S E}^{2}$ only depend on the network topology and physical parameters (as $\eta_{P M D}$ does), they can be pre-computed for a fast $Q$-factor estimation. Other noise terms such as the receiver thermal noise and shot noise are limited compared to the dominant ASE noise generated in such transmission distances, and therefore disregarded here. We also model node crosstalk as a noise variance affecting " 1 " and " 0 " bits according to [5] via the quantities $\sigma_{1, X T}^{2}$ and $\sigma_{0, X T}^{2}$. The XPM effect is modeled according to [21] and included in $\sigma_{1}$ via $\sigma_{1, X P M}^{2}$. Similarly the FWM effect is modeled as indicated by [22], [23] and is 
accounted for within $\sigma_{1}^{2}$ via $\sigma_{1, F W M}^{2}$. We refer the reader to [5], [10], [19] - [23] for additional details about the modeling of each physical impairment.

\section{GlobAl OptIMIZATION APPROACH}

This section describes the global search algorithm proposed. It is based on an iterative search of PLIA-solutions, in which each iteration rearranges parts of the lightpath demand carried through a BILP approach.

\section{A. Definitions}

Let $N$ be the set of nodes in the network, and $M$ the set of unidirectional links. We denote as |.| the number of elements of a set. The same spectral grid of $W$ wavelengths is considered in all the fibers. We define the set $S$ as $S=\{(p, w), p$ path in the physical topology, $w \in W$. Each element in $S$ is a path and wavelength assignment. We denote as $Q^{*}(p, w)$ the $Q$ factor of a lightpath occupying path $p$ in wavelength $w$, considering only static impairments. That is, in the absence of other lightpaths in the networks which could degrade its signal quality. This is calculated by calling the $Q$-tool function with a virtual topology composed of only that lightpath. We are only interested in those $(p, w)$ pairs which could be PLIA-valid, that is, for which $Q^{*}(p, w)$ is above a $Q_{\text {Threshold }}$ value corresponding to the maximum tolerable BER as defined by the network designer. We denote as $S_{I A}=\left\{(p, w), p\right.$ path, $w \in W, Q^{*}(p, w) \geq$ $\left.Q_{\text {Threshold }}\right\}$. Note that for the physical topologies of interest, significantly affected by impairment constraints, the number of elements in $S_{I A}$ is relatively low. Furthermore, $\left|S_{I A}\right|$ grows linearly with the number of links in the network, as the impairments commonly end up limiting the maximum number of hops and the maximum distance of the paths.

We denote $S_{I A}\{m\}, m \in M$ as the set of PLIA-valid $(p, w)$ pairs which traverse link $m$. We denote $S_{\mathrm{IA}}\{i \rightarrow j\}, i, j \in N$ as the set of $(p, w)$ pairs corresponding to paths initiated in node $i$, and ending at node $j$.

The lightpath demand is defined by the lightpath demand matrix $T=\left\{T_{i, j}, i, j=1 \ldots|N|\right\}$, being $T_{i j}$ the number of lightpaths to be established from node $i$ to node $j$. We define a virtual topology $V$, as a set of lightpaths established and their routes, where each lightpath is subject to the wavelength continuity constraint. Thus, a lightpath is defined by its route $p$ and its wavelength $w$. Given a virtual topology $V$, containing the lightpath $(p, w)$ we denote $Q_{\mathrm{ev}}((p, w), V)$ as the $Q$-factor of the lighptath $(p, w)$, evaluated in the presence of the other lightpaths in the virtual topology $V$, and thus considering both static and dynamic impairments.

We define the degradation matrix $D$, as a $\left(\left|S_{I A}\right| \times\left|S_{I A}\right|\right)$ matrix composed of one row and one column for each PLIAvalid $(p, w)$. Given two lightpaths, $h_{1}=\left(p_{1}, w_{1}\right), h_{2}=\left(p_{2}, w_{2}\right)$, a degradation value $D\left(h_{1}, h_{2}\right)$ measures the degradation caused in $h_{1}$, when lightpath $h_{2}$ is established, assuming that no other lightpaths apart from $h_{1}$ and $h_{2}$ occupy the network:

$$
D\left(h_{1}, h_{2}\right)=Q^{*}\left(h_{1}\right)-Q_{e v}\left(h_{1}, V=\left\{h_{1}, h_{2}\right\}\right)
$$

Of course, this measure has sense only if both lightpaths are not simultaneously unfeasible because of wavelength clashing. Note also that this degradation measure does not include effects like four-wave mixing, which appear in the presence of more than two lightpaths.

\section{B. Algorithm overview}

The algorithm is organized in 4 sequential phases. Fig. 1 includes a pseudocode describing the overall phases. Three different types of Binary Integer Linear Programming (BILP) formulations are applied in different phases of the algorithm. We denote them BILP-1, BILP-2 and BILP-3.

Phase 1 is a preprocessing stage, where $S_{I A}$ set and degradation matrix $D$ are calculated. Both calculations depend only on the physical topology (e.g. do not depend on the traffic demand). Note that computing all the possible paths in the network is not needed, but only the PLIA-valid paths. As mentioned before, this implies a relatively low number of different $(p, w)$ pairs in typical backbone networks. Regarding the $D$ matrix, the potential number of values to calculate grows with the square of $\left|S_{I A}\right|$. Fortunately, only a small subset of coordinates in the matrix have significant values. For the cases studied, a relevant degradation appears only between lightpaths sharing at least one node (which includes the lightpaths sharing one link), and with a difference in the wavelength index of at most 2 . This reduces the calculations to practical values.

Phase 2 searches for the feasible solution which carries the maximum number of lightpaths, without considering dynamic impairment constraints, but just wavelength clashing. It is conducted by solving BILP-1 formulation. The QoT of the lightpaths in the virtual topology found $V$ is evaluated invoking the $Q$-tool function. Let $V_{\text {best }}$ be the subset of lightpaths evaluated to be over the signal quality threshold. Along the algorithm, $V_{\text {best }}$ will store the $Q$-feasible virtual topology found at this moment with the maximum number of lightpaths. This defines an upper bound $\left(L_{u b}\right)$ and a lower bound $\left(L_{l b}\right)$ to the number of lightpaths of the optimum $Q$ feasible virtual topology: $L_{u b}=|V|, L_{l b}=\left|V_{\text {best }}\right|$.

Phase 3 and 4 try to find solutions with an improved number of $Q$-feasible lightpaths. This is done by searching solutions in which the number of established lightpaths is forced to be exactly $L$, for different values of $L$. For a given $L$ value, the CoreAlgorithm module is executed, which returns the best virtual topology found $V$, carrying $L$ lightpaths, and its $Q$-factor evaluation vector $Q_{v}$. This vector contains the $Q$ factor values of the lightpaths in the virtual topology. The details of the CoreAlgorithm module will be described later in this section. The $L$ values should be selected carefully: increasing values of $L$ yield to solutions with more lightpaths established, that may interfere each other and decrease the number of $Q$-feasible lightpaths.

During phase 3, solutions are searched for decreasing values of $L$. Each phase 3 iteration, searches for a solution with an $L$ value in the middle of the range $\left[L_{\text {last }}, L_{l b}\right]$, where 
$L_{\text {last }}$ is the $L$ value tested in the last iteration of phase 3 , and $L_{l b}$

Global Search Algorithm overview
Phase 1: Preprocessing
Calculate the $S_{I A}$ set.
Calculate the path degradation matrix $D$.
Phase 2: Find the first feasible solution
$V=$ BILP-1(physical topology, $\left.T, S_{I A}\right)$
$Q_{v}=Q$-tool $(V)$
$/ /$ extract the Q-feasible lightpaths from $\mathrm{V}$
$V_{b e s t}=\left\{(p, w)\right.$ in $\left.V, Q_{v}(p, w) \geq Q_{\text {Threshold }}\right\}$
$L_{l b}=\left|V_{b e s t} ; L_{u b}=\right| V \mid$.

\section{Phase 3: Down search of improving IA solution}

$L=L_{u b}$

init loop phase 3 :

$L=$ floor $\left(\left(L+L_{l b}\right) / 2\right)$

if $\left(L_{l b} \geq L\right)$

goto phase 4

end

$\left[V, Q_{v}\right]=$ CoreAlgorithm $\left(L, S_{I A}, D\right)$

$V_{\text {PLIA }}=\left\{(p, w)\right.$ in $\left.V, Q_{v}(p, w) \geq Q_{\text {Threshold }}\right\}$

add $L$ to the list of $L$ values tested

if $\left|V_{P L I A}\right|>\left|V_{\text {best }}\right| / /$ improving solution

$V_{\text {best }}=V_{\text {PLIA }}$

end

$L_{l b}=\max \left(L_{l b},\left|V_{\text {PLIA }}\right|\right)$

goto init loop phase 3

Phase 4: Up search of optimum IA solution

$L=L_{l b}+1$

$G_{L}=\min \left(10, \max \left(5\right.\right.$, floor $\left.\left.\left(\left(L_{u b}-L_{l b}\right) / 10\right]\right)\right)$

unimprLvalues $=0$;

init loop phase 4:

while $L$ value already tested in phase 3

$$
L=L+1
$$

end

$\left[V, Q_{v}\right]=$ CoreAlgorithm $\left(L, S_{I A}, D\right)$

add $L$ to the list of tested $L$ values

$V_{P L I A}=\left\{(p, w)\right.$ in $\left.V, Q_{v}(p, w) \geq Q_{\text {Threshold }}\right\}$

if $\left|V_{P L I A}\right|>\left|V_{\text {best }}\right| / /$ improving solution

$V_{\text {best }}=V_{\text {PLIA }}$

unimprovedLvalues $=0$;

else

unimprovedLvalues ++;

end

if unimprovedLvalues $==G_{L}$ end algorithm $/ / V_{\text {best }}$ is the best solution found else

$L=L+1$

goto init loop phase 4

end

Fig. 1. Pseudocode for the general scheme of the global search algorithm. is the number of $Q$-feasible lightpaths in the best solution found up to this moment. Phase 3 ends when the $L$ value is equal to or lower than $L_{l b}$. During phase 3, the list of $L$ values already tested are stored (in order that they are not repeated during the phase 4), and the best solution found is updated.

Phase 4 starts with an initial value of $L$ equal to the $Q$ feasible lightpaths in the best solution found, plus 1 . Its objective is try to find better solutions, increasing 1 by 1 the $L$ values to test, skipping the $L$ values that have been already tested (as they provided worse solutions in the past). The procedure stops if after $G_{L}$ consecutive tests of increasing $L$ values, the solutions found did not improve the best existing solution. $G_{L}$ stands for $L$-gap. If $G_{L}=1$, the algorithm stops if an $L$ value tested in phase 4 , does not improve the best existing solution. Intuitively, a $G_{L}$ value of 1 is logical in the sense that if no solution with $L$ lightpaths established is found with more lightpaths $Q$-feasible than our best solution, a higher value of $L$ could add more mutual degradation, and even give worse solutions. However, as the solutions space is not exhaustively searched, it happens that in some occasions better solutions are found with an $L$-gap greater than one. After some tests, we tuned the $G_{L}$ value to be one tenth of the gap between the number of $Q$-feasible lightpaths in the best solution after phase 3 (current $L_{l b}$ ), and the number of lightpaths in the virtual topology found in the phase $2\left(L_{u b}\right)$. Afterwards, $G_{L}$ is bounded to be between 5 and 10 . This has proved to be a good balance, suitable for different network load conditions.

\section{Core algorithm}

The CoreAlgorithm module is the fundamental part of the global search scheme. Given a total demand value $L$, it provides a virtual topology with exactly $L$ lightpaths, trying to optimize the number of lightpaths among $L$ that are $Q$ feasible. Fig. 2 displays a pseudocode for this algorithm.

The core algorithm starts from an initial solution calculated by BILP-2 formulation. Then, it iteratively searches for a solution improvement, with a bounded number of iterations $M_{i t}$. In each iteration, the previous virtual topology obtained is modified by using a BILP formulation (BILP-3). BILP-3 is designed to:

- Force some active $(p, w)$ pairs (existing lightpaths in the previous solution), to remain active in the next solution. This is denoted as the $S_{I A}\{$ r.a $\}$ set. The lightpaths to maintain are those whose $Q$-factor in the previous virtual topology is greater than or equal to current value of the $U_{1}$ threshold. Then, by using lower values of the $U_{1}$ threshold, we increase the number of lightpaths to maintain unchanged, and thus decrease the complexity of the underlying optimization problem.

- Force some inactive $(p, w)$ pairs to remain inactive in the next solutions. This is denoted as the $S_{I A}\{$ r.i $\}$ set. The lightpaths to maintain are those which (i) if were activated would clash with lightpaths in $S_{I A}\{$ r.a\}, or (ii) if were activated, would by its own degrade the $Q$ - 
factor of a lightpath in $S_{I A}$ (r.a) below the $U_{2}$ limit. That is, they are potentially harmful. A simplification is used, estimating at this point the $Q$-factor of a lightpath as the current $Q$-factor value minus the degradation calculated in the $D$ matrix. Note that although not exact, this estimation is used inside the heuristic to limit the solutions space adaptively, as the $U_{2}$ threshold controls the complexity of the underlying optimization problem. Higher values of $U_{2}$ imply that more $(p, w)$ pairs are considered as potentially harmful, and forced to remain inactive in next iteration.

- Force to find a solution with a minimum number $K$ of changes ( $K$ is always greater or equal to 1 ), respective to the previous iteration solution. Details about how changes between iterations are computed, are included in the BILP-3 explanation.

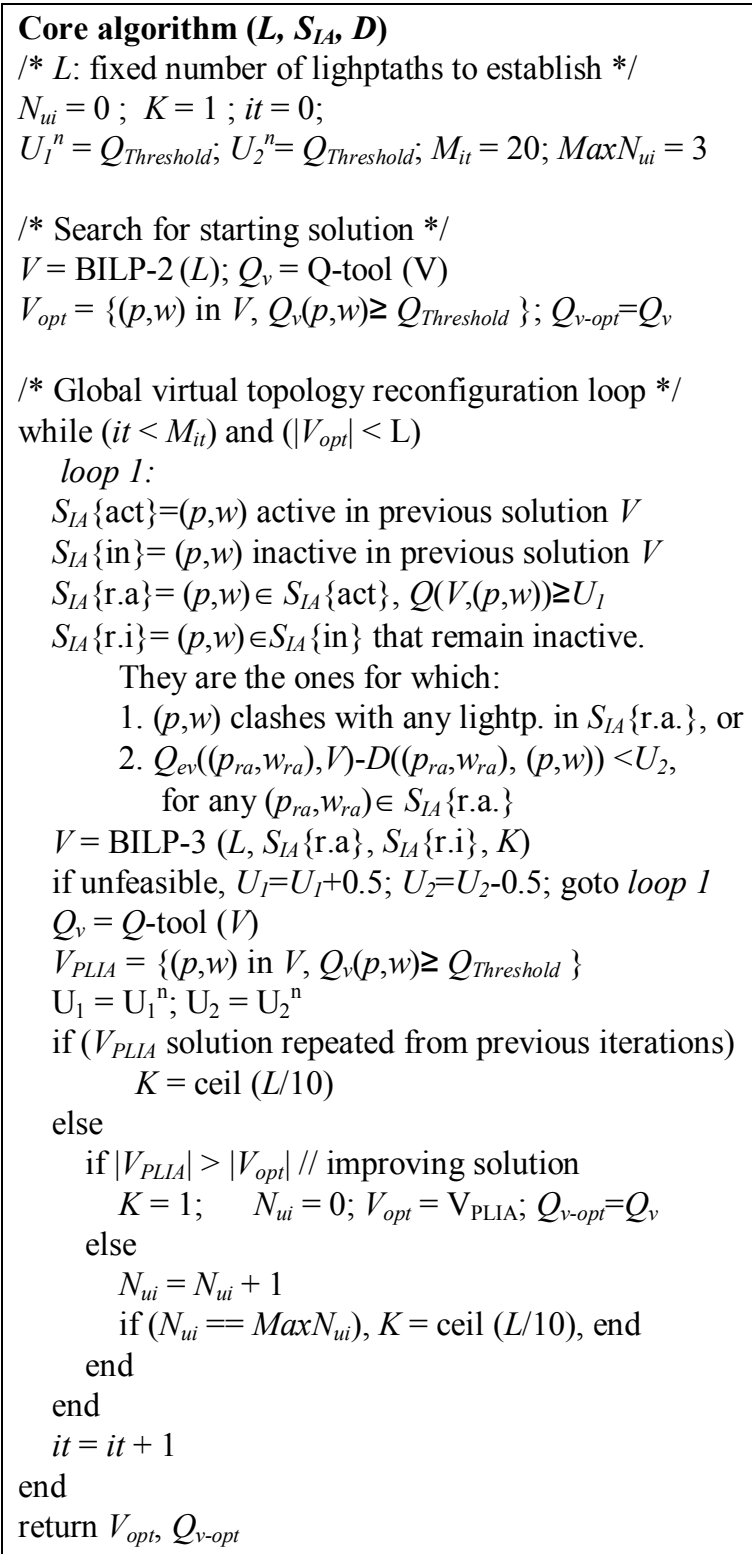

Fig. 2. Pseudocode of the CoreAlgorithm module.
The BILP-3 formulation returns a solution constrained to previous conditions. It can happen that the BILP-3 formulation cannot find a feasible solution: meeting a minimum number of changes can be impossible if at the same time the number of $(p, w)$ pairs that must remain unchanged is high. When this occurs, $U_{1}$ and $U_{2}$ thresholds are softened to reduce the number of $(p, w)$ pairs forced to be unchanged, until a feasible solution is found by BILP-3. After a solution is obtained, the best solution stored is updated, if necessary.

The algorithm includes a mechanism to avoid a large number of consecutive iterations to explore a narrow solutions space. The $K$ value is used for this purpose. When a solution is returned which is equal to one that was previously explored, the next iteration is forced to vary greatly, setting $K$ to $L / 10$, rounded to the upper integer. This means that at most $90 \%$ of the lightpaths can be left unchanged in the subsequent iteration. We can name this as a "hyper-jump" in the solutions space. Also, if the best stored solution is not improved after $\operatorname{MaxN}_{u i}$ iterations since the last "hyper-jump", one more "hyper-jump" occurs. The details about how the hyper-jump functionality is implemented are clarified in the next section.

The size of the "hyper-jump" could be modified using a simulated annealing technique, where the $K$ values are selected randomly, with the average $K$ decreasing as the number of iterations grows. This would imply a more globaloriented search in the solution space during the first algorithm iterations, which is smoothly converted into a more localoriented search along algorithm execution. This technique is left to be explored in the future.

\section{BILP formulations}

The proposed algorithm solves three different types of BILP formulations along its execution named respectively BILP-1, BILP-2 and BILP-3. All three share the decision variables, and a part of the constraints, but their objective function differ.

The decision variables in BILP-1, BILP-2 and BILP-3 are:

$x(p, w)=\{1$ path $p$ is being used by a lightpath, using wavelength $w, 0$ otherwise $\},(p, w) \in S_{\mathrm{IA}}$.

BILP-1 formulation (7) is devoted to calculate the virtual topology which maximizes the carried lightpaths, not considering impairment constraints. Constraints in $(c l)$ are the wavelength clashing constraints. They avoid that two lightpaths occupy the same wavelength in the same link. Set of constraints $(c 2)$ defines the traffic demand constraints. They limit the number of lightpaths established between two nodes, to the lightpath demand between these nodes.

$$
\begin{aligned}
& \max \sum_{p, w \in S_{I A}} x(p, w), \text { s.t. } \\
& \text { (c1) } \sum_{(p, w) \in S_{I A}\{m\}} x(p, w) \leq 1, \forall m \in M, w \in W \\
& \text { (c2) } \sum_{(p, w) \in S_{I A}\{i \rightarrow j\}} x(p, w) \leq T_{i, j}, \forall i, j \in N
\end{aligned}
$$


In the BILP-2 formulation (8), the objective is to maximize the sum of the $Q^{*}(p, w)$ values of the carried lightpaths, without considering dynamic impairments. The set of constraints $(c 1)$ and $(c 2)$ are similar to the one in BILP-1. The constrain (c3) forces to carry exactly $L$ lightpaths among the lightpath demand.

$$
\begin{aligned}
& \max \sum_{p, w \in S_{I A}} Q^{*}(p, w) x(p, w), \text { s.t. } \\
& \text { (c1),(c2) like BILP-1 } \\
& \text { (c3) } \sum_{(p, w) \in S_{I A}} x(p, w)=L
\end{aligned}
$$

In the BILP-3 formulation (9), the objective function intends to minimize the sum of the $Q$-degradation values of the solution lightpaths, over the lightpaths that are forced to remain active from previous iteration. The $Q$-degradation suffered by a lightpath $(p, w)$ is estimated as the sum of the $Q$ degradations of the other existing lightpaths over $(p, w)$. Notice that this scheme does not expect to be a way to estimate the new $Q$ factor of the lightpaths, but just a method to choose a solution which rearranges the carried demand in a way which minimizes a measure of $Q$-degradation on the lightpaths that remain unchanged. The sets of constraints ( $c 1$ c3) are the same as in BILP-2. The set of constraints (c4) forces some selected active lightpaths in previous iteration, to remain active in this iteration. The set of constraints (c5) forces some $(p, w)$ pairs which were inactive in the previous iteration, to remain inactive in the current one. Constraint ( $c \sigma)$ determines the minimum solution variation condition. This constraint forces to find a solution with a minimum number of changes $K$ in the virtual topology, respective to previous iteration. A change is accounted as the number of $(p, w)$ pairs that were active in the previous iteration, and are not active at present. Then, a lightpath reroute, a new lightpath establishment and an old lightpath tear down count as one change. This is an effective way to force large rearrangements in the virtual topology when desired, and to avoid ineffective continuous local explorations in the solutions space.

$$
\begin{aligned}
& \min \sum_{\substack{p_{1}, w_{1} \in S_{I A}\{r . a\} \\
p, w \in S_{I A}}} D\left(\left(p_{1}, w_{1}\right),(p, w)\right) x(p, w), \text { s.t. } \\
& (c 1),(c 2),(c 3) \text { like BILP-2 } \\
& (c 4) x(p, w)=1, p, w \in S_{I A}\{r . a\} \\
& (c 5) x(p, w)=0, p, w \in S_{I A}\{r . i\} \\
& (c 6) L-\sum_{(p, w) \in S_{I A}\{a c t\}} x(p, w) \geq K
\end{aligned}
$$

\section{SEQUENTIAL HEURISTIC APPROACHES}

This section describes two algorithms based on a preprocessing ordering of the traffic demand, and a subsequent sequential processing of individual lightpath demands. The algorithms are described in pseudocode in Fig. 3.
The first stage in the sequential algorithms is a demand preprocessing ordering module. We propose two different strategies to order the demands. Both are based on assessing the a-priori distance between two nodes $i$ and $j$ by the length of the shortest path between $i$ and $j$. Then, we order $(i, j)$ node pairs according to the aforementioned shortest distance, multiplied by the number of lightpaths to be established between $i$ and $j, T_{i j}$. Ties are broken randomly in this preprocessing step. Two orderings are considered: ascending and descending order. Ascending order prioritizes $(i, j)$ node pairs with shortest paths and smaller demands, while descending order prioritizes $(i, j)$ node pairs with longest paths and larger demands.

The sequential processing stage processes one by one the ordered list of $(i, j)$ pairs. For each $(i, j)$ pair, the $T_{i j}$ lightpath demands are processed sequentially. If a route and wavelength assignment is found for the lightpath demand, so that all the previously established lightpaths and the new one are $Q$ feasible, then the lightpath and its associated route are added to the current virtual topology $V$. If not, the lightpath is blocked.

A lightpath demand is processed by first finding a candidate list of possible routes and wavelength assignments for the lightpath ( $p w c$ variable in the pseudocode). For each candidate route, a candidate virtual topology $V^{\prime}$ is constructed, composed of the current virtual topology, and the new lightpath demand traversing the candidate route. $V^{\prime}$ is evaluated by means of the $Q$-tool function. If any lightpath in $V$ ' is below the QoT threshold, the candidate route is discarded. When all the candidate routes are checked, if none of them passed the QoT test the lightpath is blocked. If more than one route passes the QoT test, the route chosen is the one with the maximum worst $Q$-factor (the $Q$-factor of the lightpath with the lowest $Q$-factor).

The $p w c$ list for the node pair $(i, j)$ is constructed in our tests as follows. For each wavelength we find the $k$-shortest paths which fulfill two constraints: (i) they do not clash with existing lightpaths in $V$, (ii) they are in $S_{I A}\{i \rightarrow j\}$, which means that their static impairments do not already classify them as unfeasible paths. Note that increasing the size of the set of candidates can be controlled with parameter $k$, and has an adverse effect on the algorithm running time: all candidate routes are checked for QoT adequacy, and QoT computations are time-consuming.

\section{Simulation StUdy}

This section describes the results obtained for validation and testing of the proposed PLIA algorithms. All the algorithms have been implemented in MATLAB code, integrated and tested in the MatPlanWDM tool [24], which interfaces with the TOMLAB/CPLEX solver [25].

Firstly, we explain the assumptions about network topologies, traffic demands, and physical layer characterization considered in our simulation studies. Secondly, we introduce the simulation model employed. 
Thirdly, we expose extensive simulation results comparing the PLIA algorithms presented in this paper together with a variation of the well known LERP algorithm [26], used for comparison. Finally we discuss the results.

\section{A. Assumptions}

The algorithms have been tested for two different network topologies displayed in Fig. 4. They correspond to the Internet2 network [27] (9 nodes, 26 unidirectional links, average node degree 2.89) and the European Optical Network (EON) [28] (18 nodes, 66 unidirectional links, average node degree 3.67). Fig. 5 depicts the distribution of the distances of the shortest paths between every pair of nodes, for both

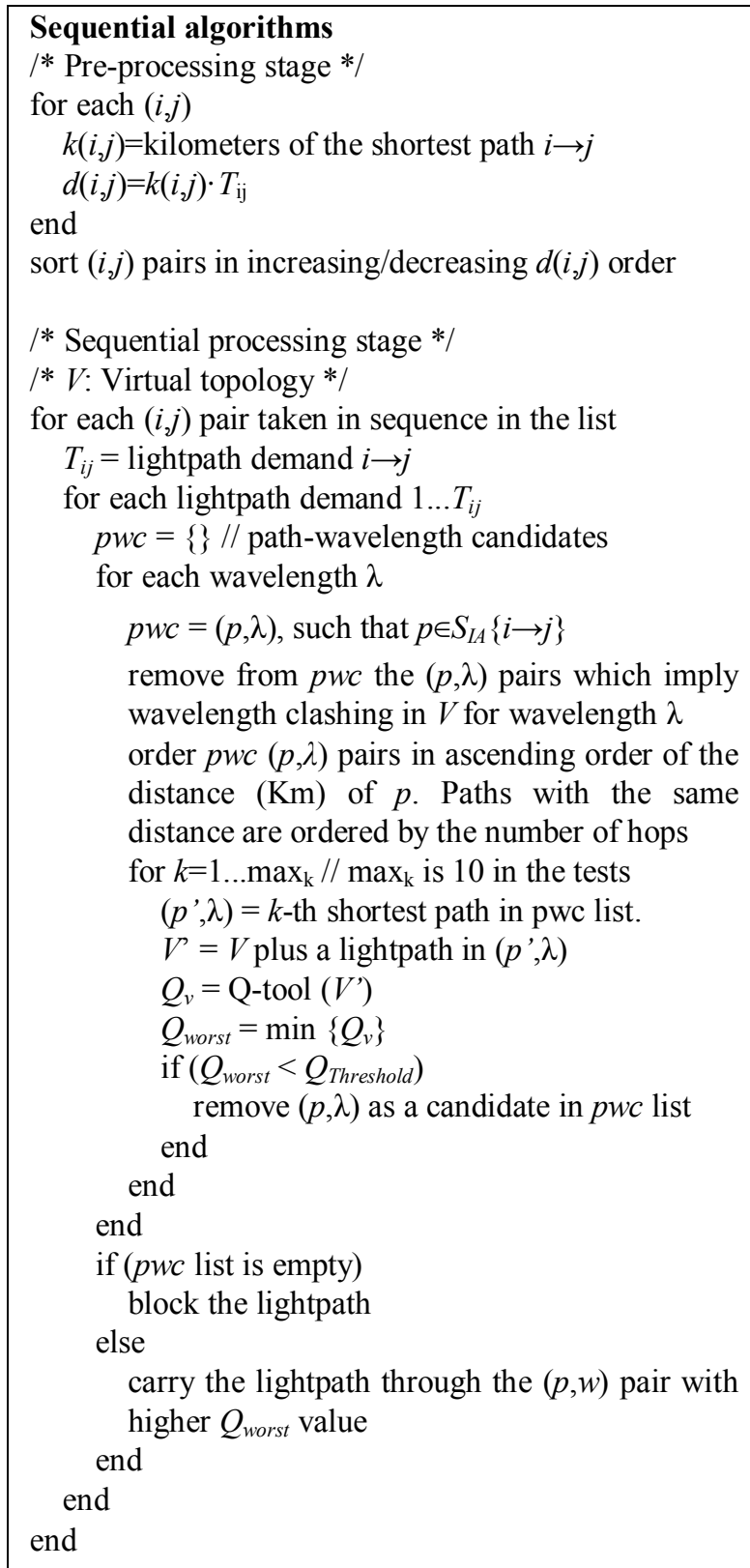

Fig. 3. Pseudocode of the sequential schemes. $\mathrm{km}$ for Internet 2 and $1203 \mathrm{~km}$ for EON topology respectively.

The traffic demand is calculated as follows. For the Internet 2 topology, a base traffic matrix was calculated from a population-distance model [29]: the traffic in Gbps between two nodes grows with the product of the population between both nodes, and decreases with the square of the distance between them. The base matrix is normalized, so that the sum of the offered traffic equals 1 Tbps. Table I shows these traffic matrix values. The base matrix for the EON topology is obtained from [28], and normalized to match a total offered traffic of 5 Tbps (Table II). Note that all the traffic matrices are symmetric. These matrices feed the performance tests shown in this section. Other results, not included in this paper, have been obtained considering asymmetric traffic matrices, which yield the same conclusions.

The PLIA algorithms proposed do not consider traffic grooming. They receive the lightpath demand as an input parameter, measured as the number of lightpaths to be established between every pair of nodes. In order to fairly evaluate the PLIA algorithms, they are tested considering a common algorithm which converts a traffic matrix (Gbps) into a lightpath demand matrix. It is based on a MILP formulation, which works as follows. First, it calculates, for every inputoutput node pair, the $Q$ factor of a lightpath in the shortest path between those nodes, considering only static impairments. If the lightpath is QoT-feasible (or $Q$-feasible), the input-output pair is considered as admissible. Then, a MILP path-flow formulation calculates the lightpath demand with the minimum number of lightpaths, which is able to carry the traffic matrix, considering only the admissible paths and an infinite number of wavelengths per fiber (that is, without considering wavelength clashing constraints).

The parameters that characterize the physical layer in all the tests for the common QoT calculation, assume a transmitter bit rate of $10 \mathrm{Gbps}$, spans of standard single mode fiber (SMF) compensated with dispersion compensation (DC) modules. The dispersion management scheme that is utilized in our studies is a pre-compensation module, to achieve better transmission reach. Every SMF span is under-compensated to a value of $30 \mathrm{ps} / \mathrm{nm} / \mathrm{km}$ in order to alleviate the non-linear effects, and the accumulated dispersion at each node becomes 0 with the use of an appropriate post-compensation module in the end of the link. The pre-compensation is set to -400 $\mathrm{ps} / \mathrm{nm} / \mathrm{km}$. Other parameters used to characterize the physical layer are the input power $(-4 \mathrm{dBm}$ at the input of the DC modules, $3 \mathrm{dBm}$ at the input of the fiber spans), grid spacing (50 GHz), type of modulation (NRZ pulses), and switch crosstalk ratio (randomly distributed between 32 and $38 \mathrm{~dB}$ ). The span length of the physical links (fibers) are set at $80 \mathrm{~km}$. The value of PMD parameter is $0.1 \mathrm{ps} / \sqrt{\mathrm{km}}$ and the fiber attenuation is $0.25 \mathrm{~dB} / \mathrm{km}$. We set $Q_{\text {Threshold }}=15.5 \mathrm{~dB}$, corresponding to a BER of $10^{-9}$ when no Forward Error Correction (FEC) is utilized. 


\section{B. Simulation results}

We have conducted two experiments to compare the performance of the proposed approaches. In the first experiment, the blocking rate (percentage of blocked lightpath demands versus total number of offered lightpath demands) is evaluated for each physical topology (Internet2 and EON) considering 8 and 16 wavelengths per fiber (Figs. 6-7). The graphs illustrate the evolution of the blocking rate, varying the traffic load. The traffic matrices used as input parameters in each simulation point, are the ones shown in Table I and II (Internet2 and EON respectively), normalized to match the value of total offered traffic, shown in the horizontal axis.

Each plot in Figs. 6-7 includes five curves. Three curves correspond to the three algorithms described in this paper (global search, and the two sequential approaches: the shortest path first (SPF) and longest path first (LPF) ordering). In addition, one more algorithm, named SLERP, is added to the comparison. The sLERP algorithm is a simplification of the LERP algorithm (Lightpath Establishment and Regenerator Placement) proposed in[26]. sLERP adapts the RWA phase of the original LERP algorithm, and skips its regenerator placement phase. In sLERP algorithm, the lightpath demand is ranked randomly, producing a randomly ordered list of 3tuples ( $i$ : source node; $j$ : destination node, $s$ : number of lightpath demands between $i$ and $j$ ). The list of 3-tuples is processed one by one. For each 3 -tuple, $k$-shortest paths are computed $(k=10)$ which are again processed in order. For each path, wavelengths are assigned to the lightpath demand following a First-Fit scheme. If there are not enough path-free wavelengths in all the $k$-shortest paths for a certain $(i, j, s)$ tuple, the exceeding lightpaths are rejected. When the random sequence is processed, the QoT of the virtual topology obtained is evaluated. The algorithm stores the best solution found: that is, the one with a higher number of Q-feasible lightpaths. The algorithm continues testing random sequences until the best solution found has not been improved for ten consecutive random orderings. As in the original LERP algorithm, a Black List with the previously tested sequences is kept, to avoid evaluating twice the same demand ordering.

The fifth curve named as stat-PLIA in the graphs, is included also for comparison. It is the blocking rate of a MILP algorithm that solves the RWA problem, maximizing the number of lightpaths carried, but including only static signal impairments. That is, the solution calculated is impairment aware in the sense that the MILP is forced to assign to the lightpaths, only those paths and wavelengths for which $Q^{*}(p, w)$ is above the quality threshold. Therefore, all the lightpaths would be valid if only static impairment were considered. The stat-PLIA algorithm is employed as an upper bound to the optimum solution.

Results show that the blocking rate obtained by the global optimization and the sequential algorithms considered are similar in the smaller topology Internet2 (Fig. 6). However, the global optimization algorithm outperforms the sequential schemes in the EON topology. Both approaches are in a significant number of occasions far from the upper bound calculated, also for small topologies. No strict conclusion can be extracted from this, as the tightness of this bound cannot be calculated. Finally, the comparison between the sequential approaches shows a better behavior in most of the situations with the shortest path ordering. The sLERP algorithm exhibited the worst performance in all the tests.

The second experiment consists of an evaluation of the blocking rate when the number of wavelengths per fiber is increased from 8 up to 32 . This experiment is repeated for the Internet 2 topology and a total offered traffic of $490 \mathrm{Gbps}$, and the EON topology for a total offered traffic of $2100 \mathrm{Gbps}$. The results from this experiment are presented in Fig. 8. They can be interpreted in the same way as in previous graphs. The performance of the sequential algorithms is similar to the global search in Internet2. For EON, a significant performance gap appears favoring the global search algorithm. This performance gap is slightly decreasing for a growing number of wavelengths per fiber.

An important conclusion can be drawn from the results in the sequential approaches. Initially, the size of the set of candidates could be seen as a tuning parameter for the tradeoff between blocking probability and running time. The parameter $k$ in the $k$-shortest path search controls this aspect. Nevertheless, our results are calculated for a value of $k=10$. This implied that, in all the cases tested, given a lightpath demand, all the possible paths which could carry a $Q$-feasible lightpath were tested. This prioritizes the lightpaths which are up in the sequence even more strongly, as they are rejected only if previous lightpaths eliminate any feasible route for the new one. However, our results show that the blocking rate performance of the sequential approaches with this exhaustive search was lower than the one of the global search, which also required a much lower number of QoT evaluations. Therefore, the results suggest that more complex sequencing strategies are needed, combined with processing schemes which allow the rerouting of existing lightpaths.

\section{SCALABILITY STUDY OF THE ALGORITHMS}

This section is aimed at discussing the scalability of the proposed algorithms, attending to the evolution of the running time of the different tests presented in the previous section. In Fig. 9 the running times of the algorithms are compared when the traffic load is increased from the $10 \%$ of the maximum load to the $100 \%$ for the four simulation scenarios (EON and Internet 2,8 and 16 wavelengths).

In the global search algorithm, the execution time is quite small for low loads, and has an abrupt rise at medium to high loads. This is because for low loads the algorithm can find a solution which carries all the traffic demand during its phase 1. For medium and highs loads the algorithm enters in the iterations inside phases 2 and 3, which are more time consuming. It is quite noticeable that at this moment, algorithm response time remains approximately constant, independent from the traffic load, the number of wavelengths, and the topology size. The small unpredictable variations 
observed are based on the particular conditions that define the algorithm stop.

Regarding also to the global search algorithm, it is relevant to know which part of the response time of the global optimization algorithm, is caused by the BILP sub-algorithms execution, and which part is caused by the QoT evaluation function. Our results are quite clear in this aspect: the average execution times of BILP sub-algorithms in the tests performed are below one second for every traffic and topology situation tested, a negligible value in static planning. Some other tests not included in this paper, for larger topologies (30 nodes) and with a higher number of wavelengths per fiber (80), also reveal a negligible contribution of the BILP executions to the total execution time. Thus, these results validate our assert that the algorithm designed effectively limits the inherent complexity problem in BILP formulations, by an appropriate control in the number of changeable decision variables.

In the end, close to the $100 \%$ of the execution time of both global search and sequential approaches is devoted to the QoT estimation of virtual topology candidates. Then, Fig. 9 illustrates the rationale of the better scalability of the global search algorithm. It shows that the number of $Q$-tool evaluations in the sequential schemes tested, grows with the traffic load, number of wavelengths and topology size. Internally, all of them contribute to the growth in the path candidate lists to evaluate. In contrast to this, the number of QoT evaluations of the global search approach depends mainly on the number of iterations performed inside each CoreAlgorithm module. The good results obtained with the small constant value of 20 iterations proves the efficiency of the solutions space exploration. Furthermore, the iterative operation of the global search algorithm opens the way to optimization strategies that stop the search after a given execution time, returning the best solution found. That time based stop procedures are not natural for sequential schemes.

\section{CONCLUSIONS}

This paper proposes and compares a set of offline algorithms for the PLIA-RWA planning problem, that make use of a common QoT evaluation function $Q$-tool, also developed by the authors. The global search approach is based on an iterative method which tries to effectively explore the solutions space. Changes between iterations are controlled by a set of BILP formulations, which favor limited rearrangements in the virtual topology searching for better solutions. The size of the rearrangement is controlled, limiting the BILP complexity. Also, the method allows to force large rearrangements between consecutive iterations to avoid a continuous local exploration of the solutions space. Both sequential and global search approaches showed a similar performance in small topologies. However, results show that this global exploration algorithm outperforms the sequential schemes tested in larger topologies. Furthermore, the time complexity of the global search algorithm remains low, and approximately constant with network size and traffic demand. The impact of BILP executions on the total execution time is negligible, in comparison to the running time of the QoT evaluation function. It can be concluded that the global search method proposed is both effective and scalable. In contrast the sequential approaches are penalized by the excessive utilization of time-consuming QoT evaluations. Results suggest that sequential algorithms should combine rerouting techniques to increase their blocking rate performance.

\section{REFERENCES}

[1] J. Berthold, A. A. M. Saleh, L. Blair, and J. M. Simmons, "Optical Networking: Past, Present, and Future," IEEE Journal of Lightwave Technology, vol. 26, no. 9, pp.1104-1118, May 2008.

[2] S. Sygletos, I. Tomkos, and J. Leuthold, "Technological challenges on the road toward transparent networking," OSA Journal of Optical Networking, vol. 7, no. 4, pp. 321-350, Apr. 2008.

[3] H. Zang, J. P. Jue, and B. Mukherjee, "A Review of Routing and Wavelength Assignment Approaches for Wavelength-Routed Optical WDM Networks," Optical Network Magazine, pp. 47-59, Jan. 2000.

[4] R. Ramaswami, and K. N. Sivarajan, "Routing and Wavelength assignment in all-Optical networks", IEEE/ACM Transactions on Networking, vol. 5, no. 3, pp.489-500, Oct. 1995.

[5] B. Ramamurthy, D. Datta, H. Feng, J. P. Heritage, and B.Mukherjee, "Impact of transmission impairments on the teletraffic performance of wavelength-routed optical networks," IEEE Journal of Lightwave Technology, vol. 17, no. 10, pp. 1713-1723, Oct. 1999.

[6] H. Yurong, J. P. Heritage, B. Mukherjee, "Connection Provisioning With Transmission Impairment Consideration in Optical WDM Networks With High-Speed Channels," IEEE Journal of Lightwave Technology, vol. 23, no. 3, pp. 982-993, Mar. 2005.

[7] S. Rai, B. Mukherjee, C.-F. Su, T. Hamada, "Provisioning in UltraLong-Haul Optical Networks," in Proc. of the Optical Fiber Communication Conference and Exposition and The National Fiber Optic Engineers Conference, Anaheim, U.S.A., Mar. 2007.

[8] Y. Pointurier, M. Brandt-Pearce, Suresh Subramaniam, and Bo $\mathrm{Xu}$, "Cross-Layer Adaptive Routing and Wavelength Assignment in AllOptical Networks", IEEE Journal on Selected Areas in Communications, vol. 26, no. 6, pp. 32-44, Aug. 2008.

[9] S. Azodolmolky, M. Klinkowski, E. Marin, D. Careglio, J. Solé Pareta, I. Tomkos, "A Survey on Physical Layer Impairments Aware Routing and Wavelength Assignment Algorithms in Optical Networks," Computer Networks (Elsevier), to be published.

[10] G. P. Agrawal, Fiber-optic communications systems, 3rd ed. New York:, John Wiley \& Sons, Inc., 2002.

[11] I. Tomkos, D. Vogiatzis, C. Mas, I. Zacharopoulos, A. Tzanakaki, and E. Varvarigos, "Performance engineering of metropolitan area optical networks through impairment constraint routing," IEEE Communications Magazine, vol.42, no.8, Aug. 2004.

[12] P. Kulkarni, A. Tzanakaki, C. Mas Machuka, and I. Tomkos, "Benefits of Q-factor based routing in WDM metro networks," in Proc. European Conference Optical Communications, Glasgow, U.K., 2005, vol. 4, pp. 981- 982.

[13] G. Markidis, S. Sygletos, A. Tzanakaki, and I. Tomkos, "Impairment Aware Based Routing and Wavelength Assignment in Transparent Long Haul Networks," in Proc. IFIP Int. Conf. Optical Network Design and Modelling, Athens, Greece, 2007, pp. 48-57.

[14] A.M. Hamad, and A.E. Kamal, "Routing and wavelength assignment with power aware multicasting in WDM networks," in Proc. Int. Conf Broadband Networks, Boston, MA, 2005, vol.1, pp. 31-40.

[15] A. Szodenyi, S. Zsigmond, B. Megyer, and T. Cinkler, "Design of traffic grooming optical virtual private networks obeying physical limitations," in Proc. IFIP/IEEE Int. Conf. Wireless and Optical Communications Networks, Dubai, United Arab Emirates, 2005, pp. 221-225.

[16] F. Cugini, F. Paolucci, L. Valcarenghi, and P. Castoldi, "Implementing a Path Computation Element (PCE) to encompass physical impairments in transparent networks," in Proc. Optical Fiber Communication Conf. and Exposition and Nat. Fiber Optic Engineers Conf. Anaheim, CA, 2007, pp. 1-3. 
[17] A. Morea, N. Brogard, F. Leplingard, J.C. Antona, T. Zami, B. Lavigne, and D. Bayart, "QoT function and $\mathrm{A}^{*}$ routing an optimized combination for connection search in translucent networks," OSA Journal of Optical Networking, vol. 7, no. 1, pp. 42-61, Jan. 2008.

[18] S. Pachnicke, T. Paschenda, and P. Krummrich, "Assessment of a constraint-based routing algorithm for translucent $10 \mathrm{Gbits} / \mathrm{s}$ DWDM networks considering fiber nonlinearities", OSA Journal of Optical Networking, Vol. 7, No. 4, pp. 365-377, April 2008.

[19] S. Norimatsu and M. Maruoka, "Accurate Q-factor estimation of optically amplified systems in the presence of waveform distortion," IEEE Journal of Lightwave Technology, vol. 20, no. 1, Jan. 2002.

[20] C. D. Cantrell, "Transparent optical metropolitan-area networks," in Proc. $16^{\text {th }}$ Annual Meeting IEEE/Laser Electro Optics Soceiety, Tucson, AZ, ,2003, vol. 2, pp. 608-609.

[21] V. T. Cartaxo, "Cross-phase modulation in intensity modulation-direct detection WDM systems with multiple optical amplifiers and dispersion compensators," IEEE Journal of Lightwave Technology, vol. 17, no. 2, pp. 178-190, Feb. 1999.

[22] W. Zeiler, F. Di Pasquale, P. Bayvel, and J. E. Midwinter, "Modelling of four-wave mixing and gain peaking in amplified WDM optical communication systems and networks," IEEE Journal of Lightwave Technology, vol. 14, no. 9, pp. 1933-1942, Sep. 1996.

[23] K. Inoue, K. Nakanishi, and K. Oda, "Crosstalk and power penalty due to fiber four-wave mixing in multichannels transmissions," IEEE Journal of Lightwave Technology, vol. 12, no. 8, pp. 1423-1439, Aug. 1996.

[24] P. Pavon-Mariño, R. Aparicio-Pardo, G. Moreno-Muñoz, J. GarciaHaro, J. Veiga-Gontan, "MatPlanWDM: An educational tool for

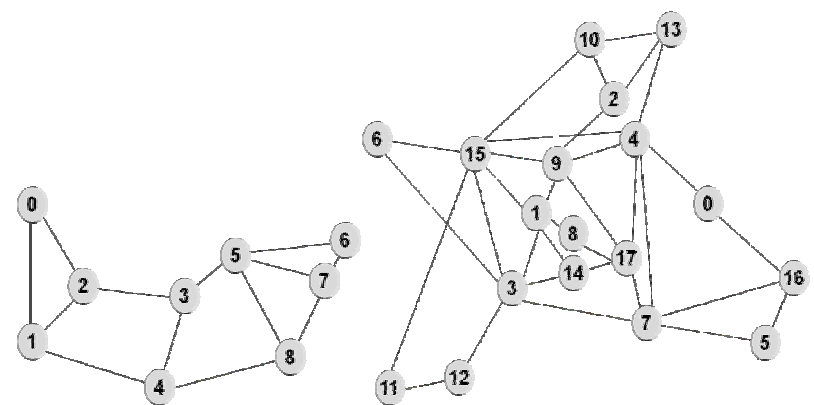

Fig. 4. Internet2 (left side) and EON (right side) physical topologies. network planning in wavelength-routing networks", Proc. IFIP Int. Conf. Optical Network Design and Modelling, Athens, Greece, 2007, pp. 58-67.

[25] TOMLAB Optimization. Available: http://tomopt.com/

[26] M. A. Ezzahdi, S. Al Zahr, M. Koubaa, N. Puech and M. Gagnaire, "LERP: a Quality of Transmission Dependent Heuristic for Routing and Wavelength Assignment in Hybrid WDM Networks", in Proc. Int. Conf. Computer Communications and Networks, Arlington, VA, 2006, pp. $125-136$.

[27] Internet 2 Global Research Network Operations Center, Web Site, [Online], Available: http://www.abilene.iu.edu/

[28] L. Wuttisittikulkij, M.J. O'Mahony, "Design of a WDM network using a multiple ring approach", in Proc. IEEE Global Telecommunications Conference, Phoenix, AZ, 1997, vol. 1, pp. 551-555.

[29] R.S. Cahn, Wide Area Network design. Concepts and tools for optimization, San Francisco, CA: Morgan Kaufmann Publishers Inc., 1998

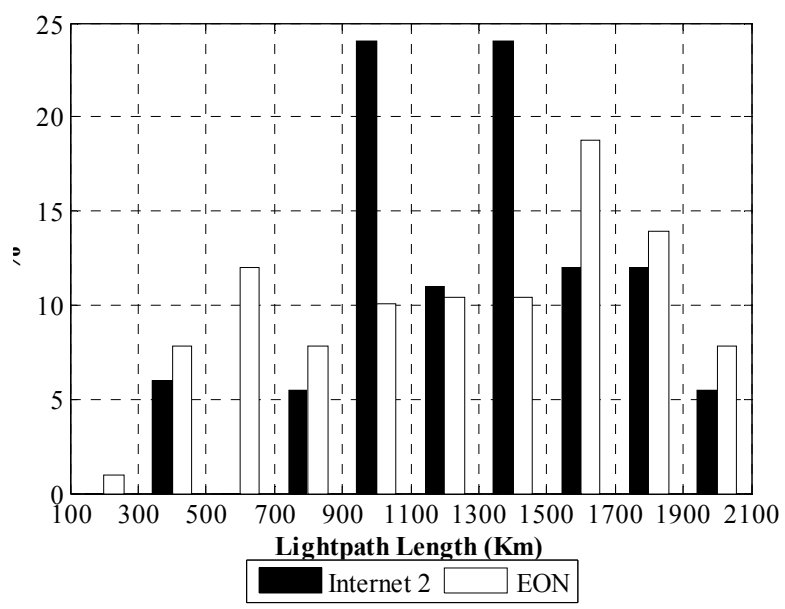

Fig. 5. Lightpath length distribution for all-pair connection demand set.

TABLE I

INTERNET2 TRAFFiC MATRIX IN GBPS (UPPER TRIANGULAR PART) AND LINK DistanCES In KM (LOWER TRIANGUL AR PART)

\begin{tabular}{c|c|c|c|c|c|c|c|c|c}
\hline & 0 & 1 & 2 & 3 & 4 & 5 & 6 & 7 & 8 \\
\hline 0 & & 16.1 & 13.8 & 8.7 & 8.5 & 8.5 & 7.4 & 6.2 & 7.0 \\
\hline 1 & 1342 & & 16.3 & 10.9 & 14.2 & 14.2 & 16.5 & 8.9 & 10.5 \\
\hline 2 & 913 & 1303 & & 11.7 & 11.2 & 10.1 & 7.5 & 7.5 & 8.8 \\
\hline 3 & - & 1330 & - & & 16.0 & 17.8 & 12.9 & 12.2 & 14.8 \\
\hline 4 & - & 1705 & - & 818 & & 16.9 & 16.2 & 12.4 & 16.3 \\
\hline 5 & - & - & - & 690 & - & & 29.5 & 18.6 & 20.0 \\
\hline 6 & - & - & - & - & - & 1400 & & 30.5 & 22.2 \\
\hline 7 & - & - & - & - & - & 905 & 278 & & 17.7 \\
\hline 8 & - & - & - & - & 1385 & 1045 & - & 700 & \\
\hline
\end{tabular}


TABLE II

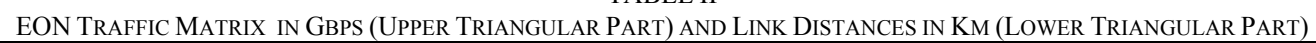

\begin{tabular}{c|c|c|c|c|c|c|c|c|c|c|c|c|c|c|c|c|c|c}
\hline & 0 & 1 & 2 & 3 & 4 & 5 & 6 & 7 & 8 & 9 & 10 & 11 & 12 & 13 & 14 & 15 & 16 & 17 \\
\hline 0 & & 8.5 & 8.5 & 17.0 & 76.5 & 8.5 & 8.5 & 42.5 & 8.5 & 8.5 & 8.5 & 8.5 & 8.5 & 8.5 & 25.5 & 17.0 & 8.5 & 8.5 \\
\hline 1 & - & & 8.5 & 51.0 & 68.0 & 8.5 & 8.5 & 17.0 & 8.5 & 34.0 & 8.5 & 8.5 & 8.5 & 8.5 & 25.5 & 34.0 & 8.5 & 8.5 \\
\hline 2 & - & - & & 8.5 & 25.5 & 8.5 & 8.5 & 8.5 & 8.5 & 8.5 & 8.5 & 8.5 & 8.5 & 8.5 & 8.5 & 8.5 & 8.5 & 8.5 \\
\hline 3 & - & 261.2 & - & & 93.5 & 8.5 & 8.5 & 42.5 & 8.5 & 42.5 & 8.5 & 8.5 & 34.0 & 17.0 & 51.0 & 85.0 & 8.5 & 8.5 \\
\hline 4 & 523.6 & - & - & - & & 17.0 & 8.5 & 76.5 & 17.0 & 68.0 & 17.0 & 8.5 & 34.0 & 51.0 & 93.5 & 68.0 & 17.0 & 17.0 \\
\hline 5 & - & - & - & - & - & & 8.5 & 17.0 & 8.5 & 8.5 & 8.5 & 8.5 & 8.5 & 8.5 & 8.5 & 8.5 & 8.5 & 8.5 \\
\hline 6 & - & - & - & 857 & - & - & & 8.5 & 8.5 & 8.5 & 8.5 & 8.5 & 8.5 & 8.5 & 8.5 & 8.5 & 8.5 & 8.5 \\
\hline 7 & - & - & - & 1102 & 735.1 & 1052 & - & & 8.5 & 17.0 & 8.5 & 8.5 & 25.5 & 8.5 & 51.0 & 25.5 & 8.5 & 8.5 \\
\hline 8 & - & 115.8 & - & - & - & - & - & - & & 8.5 & 8.5 & 8.5 & 8.5 & 8.5 & 8.5 & 8.5 & 8.5 & 8.5 \\
\hline 9 & - & 205 & 730 & - & 680 & - & - & - & - & & 8.5 & 8.5 & 17.0 & 8.5 & 25.5 & 42.5 & 8.5 & 8.5 \\
\hline 10 & - & - & 550 & - & - & - & - & - & - & - & & 8.5 & 8.5 & 8.5 & 8.5 & 8.5 & 8.5 & 8.5 \\
\hline 11 & - & - & - & - & - & - & - & - & - & - & - & & 17.0 & 8.5 & 8.5 & 8.5 & 8.5 & 8.5 \\
\hline 12 & - & - & - & 1100 & - & - & - & - & - & - & - & 583 & & 8.5 & 17.0 & 8.5 & 8.5 & 0.0 \\
\hline 13 & - & - & 561 & - & 811 & - & - & - & - & - & 418.8 & - & - & & 8.5 & 8.5 & 8.5 & 8.5 \\
\hline 14 & - & 488.7 & - & 302.9 & - & - & - & - & - & - & - & - & - & - & & 25.5 & 8.5 & 8.5 \\
\hline 15 & - & 380 & - & 484 & 934.3 & - & 570 & - & - & 401 & 1154 & 1597 & - & - & - & & 8.5 & 8.5 \\
\hline 16 & 320 & - & - & - & - & 1073 & - & 590 & - & - & - & - & - & - & - & - & & 8.5 \\
\hline 17 & - & - & - & - & 379 & - & - & 1200 & 371.2 & 714.5 & - & - & - & - & 530.4 & - & - & \\
\hline
\end{tabular}

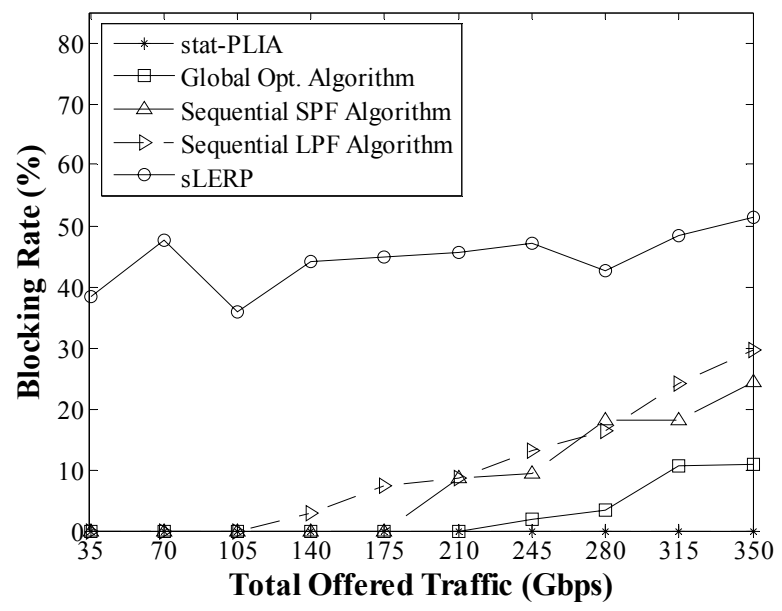

(a)

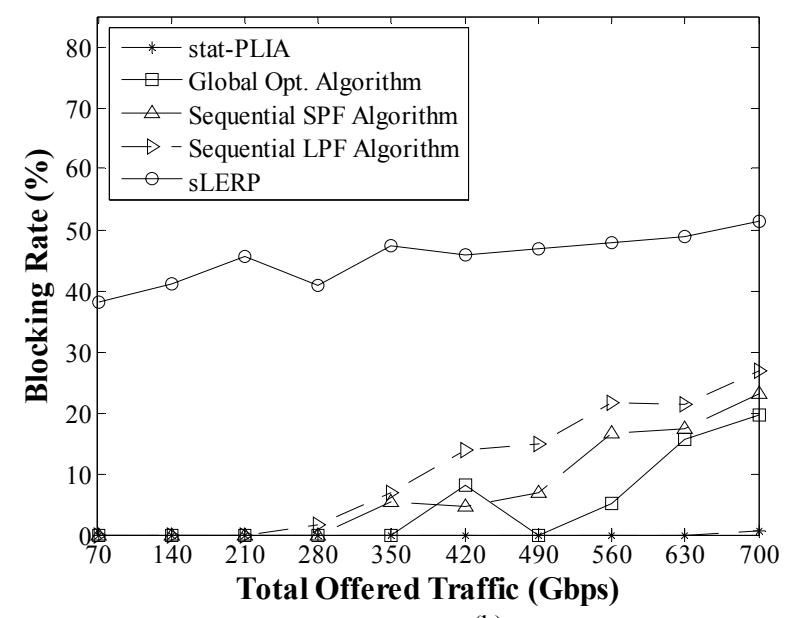

(b)

Fig. 6. (a) Blocking Rate vs. Load Internet2 8 wavelengths . (b) Blocking Rate vs. Load Internet2 16 wavelengths. 


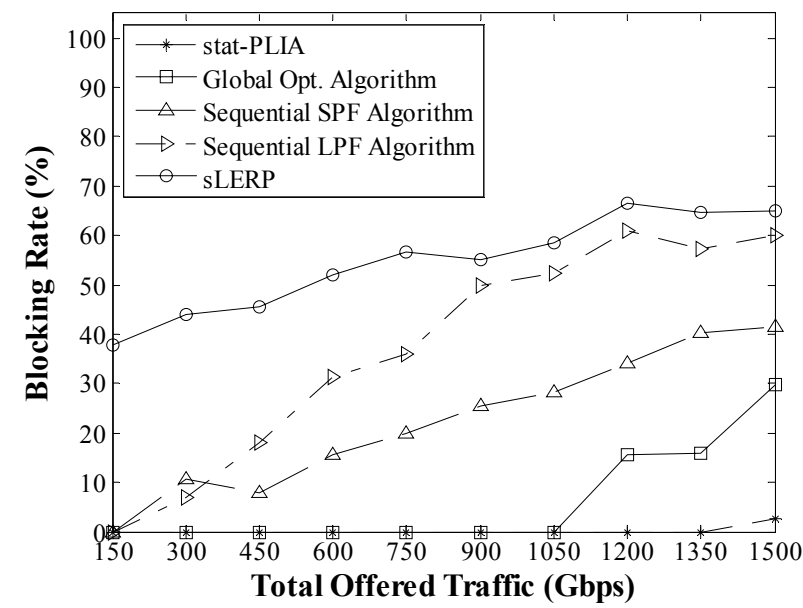

(a)

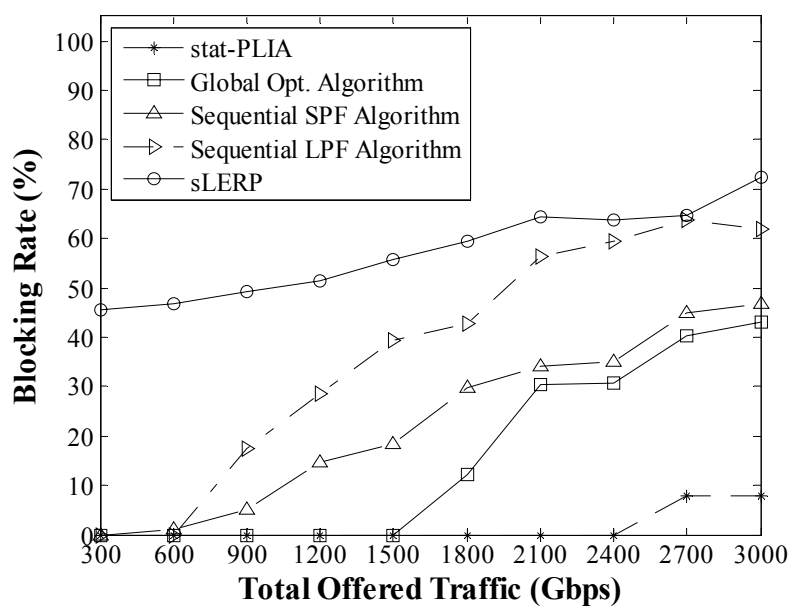

(b)

Fig. 7. (a) Blocking Rate vs. Load EON 8 wavelengths. (b) Blocking Rate vs. Load EON 16 wavelengths.

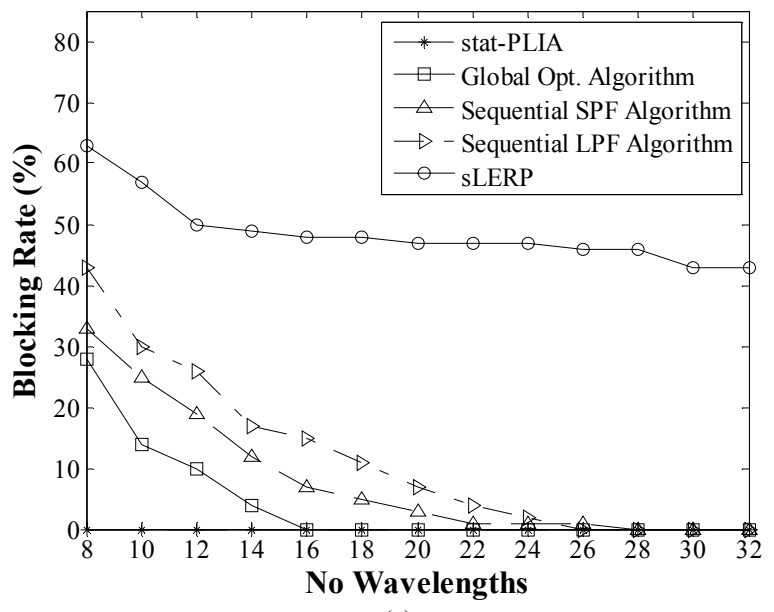

(a)

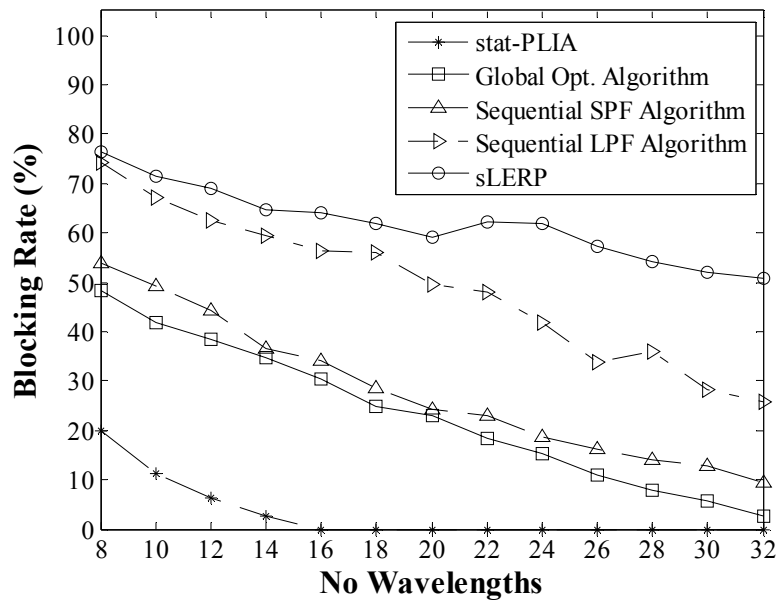

(b)

Fig. 8. (a) Blocking Rate vs. Number of wavelengths Internet2. (b) Blocking Rate vs. Number of wavelengths EON. 


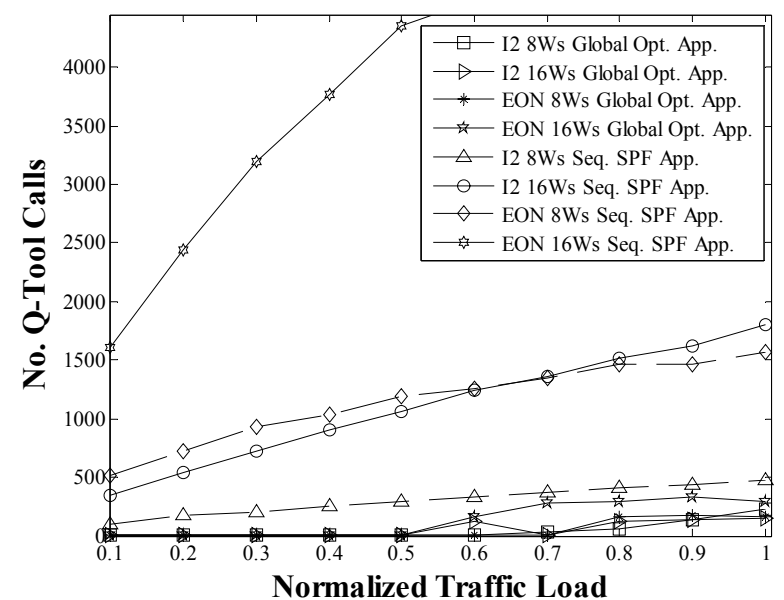

(a)

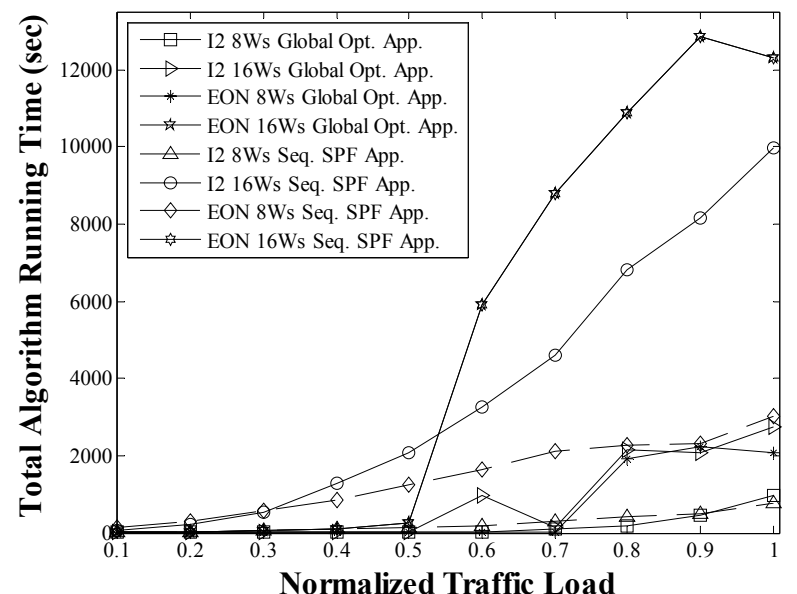

(b)

Fig. 9. (a) Number of Q-Tool Calls vs. Normalized Traffic Load. (b)Total Algorithm Running Time (sec) vs. Normalized Traffic Load. 
